\title{
Diagnostic Enigma of Spindle Cell Carcinoma of the Oral Cavity- Review of Literature
}

\section{Flora Verma, Priyanka Kamra*, Deepti Anand and Manisha Dixit}

Periodontology, CDER, AIIMS, New Delhi, India

*Corresponding Author: Priyanka Kamra, Periodontology, CDER, AIIMS, New Delhi, India.
Received: May 24, 2021

Published: June 29, 2021

(C) All rights are reserved by Flora Verma., $\boldsymbol{e t}$

al.

\begin{abstract}
Spindle cell carcinoma is a variant of squamous cell carcinoma which has been reported in oral cavity with different demographic data. Spindle cell carcinoma has been addressed with various terminologies including, "sarcomatoid carcinoma", "collision tumor", "pseudocarcinoma" owing to its varied proposed histogenesis. Spindle cell carcinoma is important to understand due to its perplexing pathological diagnosis. The "dedifferentiating" epithelial population into spindle cell morphology leads to overlapping features with other sarcomatous tumors. It is essential to evaluate the lesion and attain a proper diagnosis for better treatment and clinical outcomes. Thus, in this review published literature and reported cases has been presented to understand such aggressive lesion with importance of using diagnostic techniques such as immunohistochemistry to understand the pathogenesis.
\end{abstract}

Keywords: Squamous Cell Carcinoma; Oral Cancer; Sarcomatoid; Spindle Cells; Immunohistochemistry

\section{Introduction}

Spindle cell carcinoma (SpCC), also called sarcomatoid carcinoma or pseudosarcoma or pleomorphic carcinoma or polypoid carcinoma or carcinosarcoma is a relatively an rare tumor [1]. The W.H.O. classification of tumors of the oral cavity and oropharynx has placed this disease entity under malignant epithelial tumors of Squamous Cell Carcinoma (SCC) and labeled it "Spindle cell carcinoma" (SpCC). It is an unusual form of poorly differentiated squamous cell carcinoma (SCC) which has spindled or pleomorphic tumor cells [2]. SpCC is a biphasic tumor with divergent differentiation composed of a SCC, either in situ and/or invasive, with a malignant spindle cell component. It is a malignancy of epithelial origin often mimicking its mesenchymal counterpart [3].

These tumors pose a significant diagnostic challenge to the clinician with remarkable morphological, histopathological and immunohistochemical overlap resembling other benign and malignant spindle cell tumors $[4,5]$. There has been confusion over the basic nature of the sarcomatoid element, whether it is benign or malignant, and mesenchymal or epithelial in origin or a biphasic derivative of pseudosarcoma or a collision tumor [6]. An accurate diagnosis of these tumors is essential for proper management. This review is being carried out to provide a brief overview of the prevalence, clinical features, histopathological variants, site specificity, management and outcomes in the oral cavity based on the cases reported in literature.

\section{Prevalence and incidence}

Spindle cell carcinomas are atypical variants of squamous carcinoma accounting for $3 \%$ of all squamous carcinomas of the head and neck region [7]. These tumors are relatively uncommon in the oral cavity; reportedly accounting for less than $1 \%$ of all tumors 
of oral regions [8]. The incidence rate of spindle cell carcinoma is about 0.59 percent of all upper aerodigestive tract neoplasms.

Age and sexual predilection

Leventon., et al. reported age -range of 47 years to 88 years with a mean age of 65.7 at the time of diagnosis and a greater predilection in male patients [6]. Vishwanathan., et al. reported age-ranges from 22 - 90 years (median 53 years), and male:female ratio of 3.7:1 [9]. Feng., et al. reported that SpCC occurs in the seventh decade of life and the male-to-female ratio is almost $1: 1$ without predominance in any gender [10].

Spindle cell carcinoma of the oral cavity presents with a profound male to female predilection (11:1) and the mean age of occurrence is 57 years; although it can be diagnosed in a younger age group and very old age group (range 29 - 93 years) [11,12].

\section{Site specificity of the oral and maxillofacial region}

SpCC is a rare variant of squamous cell carcinoma and most frequently occurs in the larynx, with infrequent occurrence in various other organs like upper aerodigestive tract $[1,13,14]$ including hypopharynx and nasal cavity, esophagus, skin and breast [2]. It also show predilection for the lower lip, tongue and alveolar ridge or gingiva $[11,12]$. In order to review a summary of the cases and the site specificity in the oral cavity data have been tabulated. Table 1 presents the cases reported in the literature.

\begin{tabular}{|l|c|c|}
\hline \multicolumn{1}{|c|}{ Author } & Year & Site of lesion \\
\hline Munakata., et al. [15] & 1998 & Gingiva (retromolar area) \\
\hline Katase., et al. [16] & 2008 & $\begin{array}{c}\text { Posterior mandibular } \\
\text { gingiva }\end{array}$ \\
\hline Kwon., et al. [17] & 2010 & Mandible \\
\hline Parikh., et al. [18] & 2011 & Maxillary alveolar ridge \\
\hline Ravindran., et al. [19] & 2013 & Maxilla \\
\hline Shen., et al. [20] & 2014 & Mandibular gingiva \\
\hline Shetty., et al. [21] & 2015 & Anterior maxilla \\
\hline Mahajan., et al. [22] & 2017 & Mandible alveolar ridge \\
\hline Patnakar., et al. [23] & 2018 & $\begin{array}{c}\text { Anterior mandibular } \\
\text { gingiva }\end{array}$ \\
\hline Mathew., et al. [24] & 2019 & Maxillary alveolus \\
\hline Palla B., et al. [25] & 2020 & Anterior Maxilla \\
\hline Ono S., et al. [26] & 2021 & Tongue \\
\hline
\end{tabular}

Table 1: List of spindle cell carcinoma cases published.
Pre-disposing factors

Cancer of oral cavity has been reported to have various risk factors, including tobacco, alcohol dietary and nutritional habits as well as daily intake of macro as well as micro nutrients [27]. In spindle cell carcinoma, 4 factors are considered to be possibly associated with this disease: tobacco abuse and smoking, alcohol abuse, poor oral hygiene, and previous irradiation to the area of tumor [18,23]. Viswanathan., et al. in a clinicopathologic review of 103 cases of sarcomatoid carcinoma of the head and neck reported that tobacco chewing (63.8\%) was more frequently observed than smoking $(21.3 \%)$ in patients without any past history of radiation exposure. Where smoking tobacco is a predominant habit, the maximum number of cases were reported in the larynx [9]. Table 2 represents the commonly associated predisposing factors in the cases reported in the literature.

\begin{tabular}{|c|c|c|}
\hline Author & Year & Pre-disposing factor \\
\hline Munakata., et al. [15] & 1998 & Not known \\
\hline Katase., et al. [16] & 2008 & Not mentioned \\
\hline Kwon., et al. [17] & 2010 & Non smoker \\
\hline Parikh., et al. [18] & 2011 & Smoking \\
\hline Ravindran., et al. [19] & 2013 & Paan chewing \\
\hline Shen., et al. [20] & 2014 & Not mentioned \\
\hline Shetty., et al. [21] & 2015 & Not mentioned \\
\hline Mahajan., et al. [22] & 2017 & $\begin{array}{c}\text { No history of smoking and } \\
\text { alcohol }\end{array}$ \\
\hline Patnakar., et al. [23] & 2018 & Tobacco quid placement \\
\hline Mathew., et al. [24] & 2019 & $\begin{array}{c}\text { No history of smoking and } \\
\text { alcohol }\end{array}$ \\
\hline Palla B., et al. [25] & 2020 & Smoking \\
\hline Ono S., et al. $[26]^{26}$ & 2021 & $\begin{array}{l}\text { No history of smoking and } \\
\text { alcohol }\end{array}$ \\
\hline
\end{tabular}

Table 2: Predisposing factors for Spindle cell carcinoma of oral cavity.

\section{Onset of the disease/symptomology}

Most of the patients present a short duration of onset of less than 1 year [5]. Viswanathan., et al. reported that the duration of symptoms ranged from 20 days to 2 years with less than 1 year in $95 \%$ of patients [9]. Majority of the patients present with a hard, indurated, non-mobile, painful or painless swelling, sometimes an echymotic or non-healing ulcer which showed a rapid increase in 
size in a very short duration of time. Although swelling was a consistent finding symptoms such as regional lymphadenopathy, inability to eat properly paresthesia or numbness, inability to open the mouth, tooth mobility with angular bone less, hyperplastic gingiva or root resorption are found associated depending on the site of the lesion. Tumors in the maxilla or nasal cavity sometimes can present with excessive tearing from the eye, mass lesion, nasal blockade and bleeding $[17,18,22]$. Table 3 presents the symptomology of the reported cases.

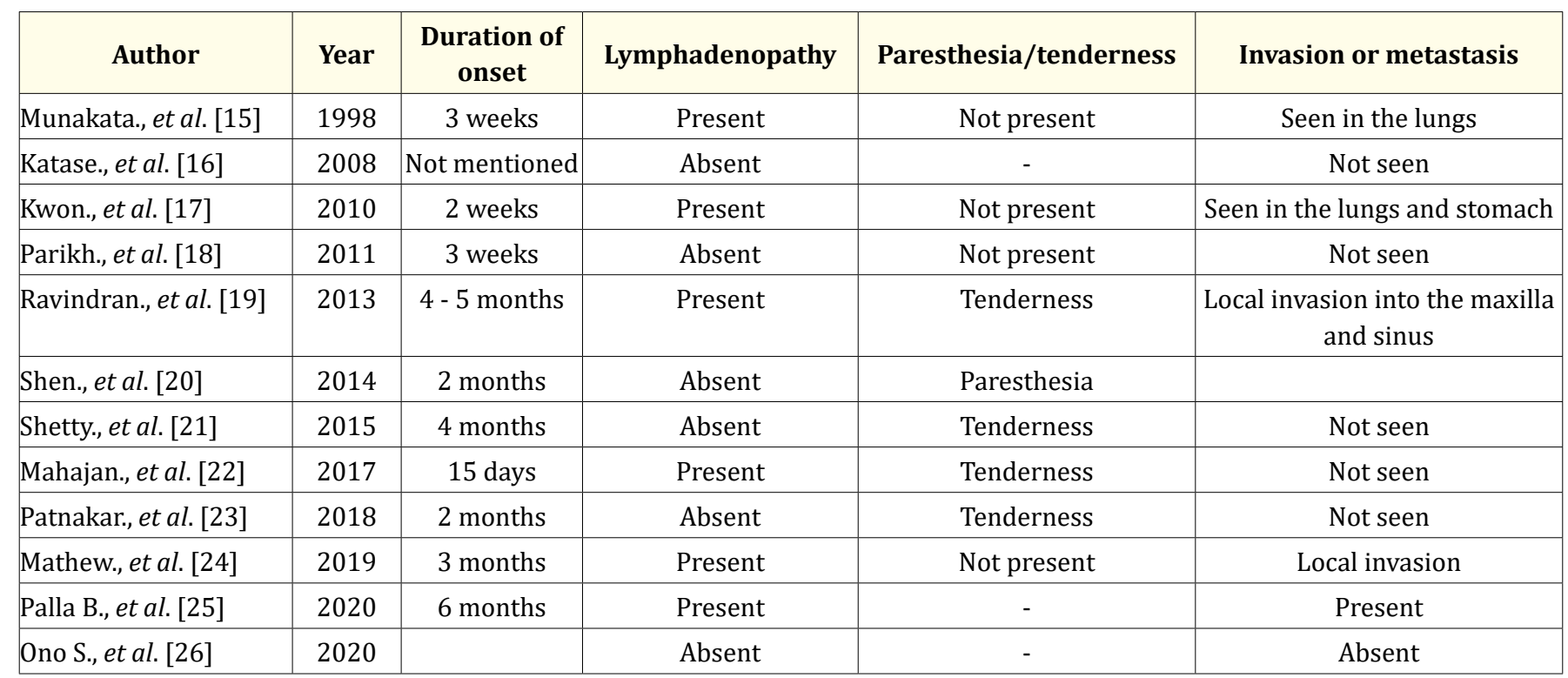

Table 3: Symptoms reported in published literature.

\section{Clinical presentation}

The tumor usually represents as large exophytic, polypoid growth of variable size $(2-6 \mathrm{~cm})$ with rough irregular ulcerated surface covered with brown necrotic tissue or with erythematous patches [9]. These ulcerative and necrotic areas are often associated with pain [25]. Although, sessile, pedunculated nodular or endophytic presentations have also been described in the literature [9].

\section{Radiographic apprearance}

Panoramic and intra-oral radiographs present a variable picture depending on the site of the tumor. An irregular radiolucency or radio-opacity with ill-defined margins, localized or diffuse bone loss with or without sclerotic margins with cortical bone invasion and destruction can be seen in the posterior mandibular lesions. The anterior mandibular lesions may reveal severe vertical bone loss with destruction of trabecular pattern, widening of periodontal space and break in the continuity of lamina dura of the incisors. Root resorption or displacement of the teeth can also be seen in some cases. The lesions of the gingiva may not present with any ra- diographic alteration. A CT scan or MRI might be required to delineate the exact dimensions, extent and invasion into the surrounding areas and to preclude any soft tissue involvement $[15,20]$.

The differential diagnosis includes osteomyelitis, pyogenic granuloma, osteonecrosis, odontogenic tumor or any metastatic tumor on the basis on radiographic presentation. Thus, a biopsy is required to confirm the provisional diagnosis [20].

\section{Gross examination}

The overlying mucosa may appear polypoidal or ulcerated while submucosa gives a firm fibrous appearance. The ulcerated areas are covered by fibrinoid material and slough [9]. Similarly, Parikh., et al. reported a case having gross polypoid growth. Cut surface was grey white in color and firm in consistency [18].

\section{Microscopy}

Microscopically the spindle cell carcinoma presents two components - epithelial component and sarcomatoid component. The 
proportion of these components varies in every case. Usually, the epithelial component is less than the sarcomatoid. It is believed that despite the epithelial and spindle components shows phenotypic divergence, but have similar tumorigenic pathway [18].

\section{Epithelial squamous component: (Carcinomatous/SCC)}

The squamous component has been reported to appear in form of dysplasia, conventional squamous carcinoma nests, squamoid differentiation, Carcinoma-in situ. Sarcomatoid and squamous component seen in complex fashion. Sometimes it is difficult to indistinct the overlying epithelium origin as the basal cells show elongating and spindling into the stromal areas $[9,18]$.

Sarcomatoid spindle component (Dysplastic spindle cells)

This component arises when the epithelial component undergoes phenotypic changes and converts into a spindle cell. These cells acquire Mesenchymal expression [18].

Sarcomatoid component can be divided based on degree of anaplasia- mild, moderate and severe anaplasia [9].

Sarcomatoid areas in spindle cell carcinomas shows varied patterns which resembles Mesenchymal malignancies histopathologically and show similar immune-expression. Tumor cells can be seen organized in fascicles, storiform pattern, areas of severe inflammation as well as granulation tissue resembling inflamed myofibroblastic tumors. Cases may show epithelioid appearing tumors cells [9]. Few authors also stated that these patterns might have a link with tumor invasion and metastasis [2].

Parikh., et al. reported a case spindle cells histologically appeared bizarre, basophilic in nature, hyperchromatic and pleomorphic (malignant histiosarcoma like) [18]. While the invading spindle cells with prominent mitotic figure, atypical mitosis were laid into fascicle pattern, resembling fibrosarcoma. These spindle cells have been reported to undergo alterations leading the keratin loss. While areas may show true metaplasia of epithelial cells into fibroblast like cells, having acquired both morphological and functional properties of a Mesenchymal cell. These metaplastic altered spindle cells results in vimentin positive [18].

\section{Tumor matrix}

The intervening stroma demonstrates presence of collagen, marked myxoid component. This matrix in different proportions along with the tumor cells forms numerous patterns [9]. Presence of areas showing adipose tissue, myxoid and necrosis has also been reported. Tumor stroma may also show evidence of other cell population such as acute and chronic inflammatory cells [25], tumor giant cells [9].

\section{Lymph node metastasis}

Morphologically it presents squamous carcinoma or mixed epithelial and spindle appearance or spindle component purely [9]. Viswanathan., et al. studies the cases by dividing then into three groups: Group I- Frank epithelial differentiation, Group II- Epithelial differentiation at IHC level only, Group III - lack of epithelial differentiation [9].

\section{Metaplastic changes}

Degree of metaplastic changes occurring in a tumor varies. Few theories have been put forward for these metaplastic changes 1) Due to stromal activation linked to human-host interface, 2) Due to radiotherapy, although it is still unclear. Along with this, it has been seen that its affection on clinical findings and prognosis metaplastic is not found [16]. Apart from this, few cases with foci of osteoid, cartilage and bone formation has been reported giving osteosarcomatous appearance $[9,26]$.

\section{Need for IHC}

To establish a diagnosis of epithelial origin especially in cases with no evidence of squamous differentiation, Immune-expression of epithelial markers plays important role. The percentage and intensity of the markers varied in different studies ranging from weak focal to diffuse and intensely positive [9].

It was noted that occasionally the Mesenchymal markers expressed aberrantly leading to diagnostic confusion. It should be noted that cases where the both epithelial differentiation epithelial markers expression is absent, diagnosis can be reached by exclusion [9]. Absence of immune-staining can be due to different factors such as improper sampling, poor fixation, sensitive IHC technique, intra-observer subjectivity less epithelial component present or only a part of biopsy is available for assessment [9].

Another point to be kept in mind is that the epithelial markers have been reported to decrease in expression and even lost completely within the tumor. This does not excludes the diagnosis of sarcomatoid carcinoma [9]. 
Most sensitive and consistent epithelial markers to confirm epithelial phenotype are keratin (AE1/AE3) and EMA (epithelial membrane antigen). Spindle cell component showing positive expression for these two markers can be helpful in the differential diagnosis of Sarcomatoid carcinoma with other sarcomatous lesions. Another important point states that in spindle cell carcinoma a positive response for vimentin in sarcomatoid cells should not be ruled out of the differential diagnosis [21].

Addition to it, a panel of anti-keratin antibodies should be used. Mere, absence of keratin staining in sarcomatoid tumor cells does not always exclude Spindle cell carcinoma as it may come positive for only some anti-keratin antibodies. Different kinds of anti-keratin antibodies should be applied in the differential diagnosis. To include wide range of cytokeratins, PAN-CK can be used [21].

Taken into account, the bizarre fibroblastic population, the spindle cells with true mesenchymal features (both morphological and functional), these show vimentin positivity. P63 is another marker used for the diagnosis [21].

A study by Ramamurti., et al. enlightened the use of IHC marker for evaluating the invasive potential in such neoplasms. For this purpose they used podoplanin, mucin like glycoprotein which is important for lymphangiogenesis. Noted that it is not a diagnostic marker but can be used to assess the potential of a tumor to spread $[8,23]$. Table 4 enlists the important antibodies that can aid in diagnosis of spindle cell carcinoma of oral cavity.

\section{Electron microscopy}

Studies have demonstrated presence of desmosomes and tonofilaments which strongly supports the epithelial origin of spindle cell component [18]. Ultrastructurally, transitional zone exists between the carcinomatous and sarcomatous components, the latter generally accounting for $>50 \%$ of the lesion [20].

\section{Differential diagnosis}

Differential diagnosis of sarcomatoid carcinoma may include other benign lesions and mesenchymal malignancies- Squamous cell carcinoma [9] mucosal spindle cell melanoma, leiomyosarcoma, myoepithelial carcinoma [17], malignant fibrous histiocytoma, rhabdomyosarcoma, malignant peripheral nerve sheath tumor, osteosarcoma, mesenchymal chondrosarcoma, malignant melanoma,

\begin{tabular}{|c|c|c|}
\hline \multirow[b]{2}{*}{ Immunomarkers } & \multicolumn{2}{|c|}{ Immunoreaction } \\
\hline & $\begin{array}{l}\text { Spindle cells } \\
\text { population }\end{array}$ & $\begin{array}{c}\text { Epithelial cell } \\
\text { population }\end{array}$ \\
\hline \multicolumn{3}{|l|}{ Cytokeratins } \\
\hline AE1/AE3 & + & + \\
\hline 8 & $+/-$ & + \\
\hline 19 & $+/-$ & + \\
\hline Cam5.2 & - & $+/-$ \\
\hline Vimentin & + & - \\
\hline S-100 & - & - \\
\hline CD 68 (KP1, PGM1) & - & - \\
\hline Pan-actin (HHF-35) & - & - \\
\hline Lysozyme & - & - \\
\hline A-Antitrypsin & + & - \\
\hline Desmin & - & - \\
\hline $\begin{array}{l}\alpha \text { SMA (alpha smooth } \\
\text { muscle actin) }\end{array}$ & $+/-$ & $+/-$ \\
\hline Osteopontin & - & - \\
\hline BMP-2 & - & - \\
\hline BMP-4 & - & - \\
\hline Ki-67 & $>40 \%$ & $<30 \%$ \\
\hline p53 & $50 \%$ & $50 \%$ \\
\hline
\end{tabular}

Table 4: Summarizing expression of immunomarkers for different tumor population $[11,21,25]$.

BMP: Bone Morphogenetic Protein; +: Positive, +/-: Focally Positive, -: Negative.

fibromatosis, leiomyoma, nodular fasciitis, reactive epithelial proliferations [9]. Literature also states that sometimes may resemble bony lesions such as osteosarcoma extending to mucosa causing diagnostic dilemma. In such cases radiographic picture should be taken into consideration [17]. Patankar., et al. also states that they should be differentiated from fibrosarcoma and true superficial sarcomas [23].

\section{Treatment}

Treatment of choice involves surgical resection of the tumor along with neck dissection [23]. 
Su., et al. observed in their study that surgical margins were negative in local recurrence cases and thus suggested wider safe margin $>2 \mathrm{~cm}$. Further, a close post-operative follow up may help to detect early recurrence [8].

Additionally, radiotherapy or chemotherapy also play a part in treatment but varies from case to case. Mostly, radiotherapy and chemotherapy is given post-surgery. This is because of adverse prognostic factors including unconfirmed surgical margins, poor differentiation pathologically and advanced tumor stage etc [10]. Adjuvant radiotherapy has also been reported to be of some help in improving local control in with positive surgical margins but cannot be relied on. Chemotherapy will still holds its benefit in the future, with distant recurrence cases. Cases with high angiogenic response and > T2 tumor staging may need chemotherapy to reduce risk of metastasis [2].

\section{Prognosis}

The biological behavior of each tumor differs. Kwon GY., et al. states that the prognosis depends on location, size of tumor, depth of invasion, stage along with presence of any keratin immunestaining in the spindle cells. The presence of such neoplasm in oral cavity or oropharynx may show aggressive behavior and reoccur often easily [17]. Spindle cell carcinoma cases show location specific prognosis [10].

Deep invasive tumors show poor prognosis while early stage cases have excellent prognosis. Some of the reliable prognostic features may include, distant metastasis, depth of tumor invasion and polypoid configuration [21]. Study by Su., et al. concluded that spindle cell carcinoma cases show 36.7 overall survival rate in one year and in early cases it was $100 \% 3$ year survival [2]. Another study stated a $55 \%$ survival rate in 2 years and $36 \%$ metastases prevalence [15]. It is identified presence of inflammatory state can cause the epithelioid cell to change to spindle phenotype to support migration. Su., et al. also highlighted that oral and oro-pharyngeal spindle cell carcinoma has more aggressive behavior, where even early stage cases can show high recurrence rate and advance stage cases have high metastatic rate [2].

Cases with histopathological evidence of osteoid, cartilage or bone formation did not yield in poor prognosis when compared cases with absence of this feature [26].
Recent case report by Palla B., et al. highlights that a noticeable difference exists between spindle cell carcinoma when compared to SCC, when prognosis and overall survival is taken into consideration. They considered only $30 \%$ of 5 year survival in cases of oral spindle cell carcinoma [25].

\section{Diagnostic criteria}

$\mathrm{Su} \mathrm{HH}$. , et al. in his study mentioned the diagnostic criteria which needs to be fulfilled for Spindle cell carcinoma. It included Identifying carcinoma with squamoid feature within or in some part of the tumor, Cytokeratin positive and vimentin negative spindle cells and presence of carcinoma in situ [2]. A diagnostic plan is explained in figure 1.

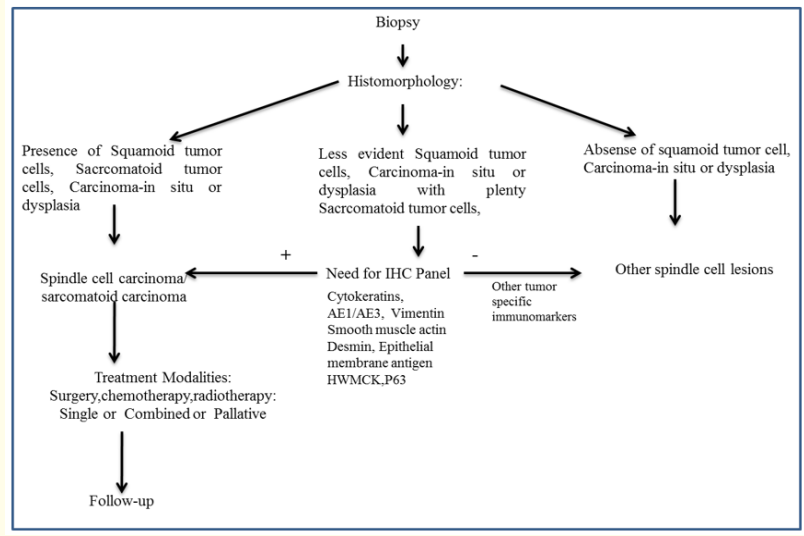

Figure 1: Diagnostic algorithm for spindle cell carcinoma of oral cavity.

\section{Conclusion}

Spindle cell carcinoma which still demands a systematic approach for an precise diagnosis and patient management. Taking into account the aggressive behavior of spindle cell carcinoma of oral and oro-pharyngeal region the treatment should be targeted to control local as well as distant recurrences. A planned management of such aggressive lesion is needed which includes clinical, radiographic evaluation but most essential is histopathological diagnosis aided with special stains and immunohistochemical staining for tumor cell population and stromal changes, that would give a better insight into the pathogenesis of spindle cell carcinoma. Prognostic indices may further help to reveal the prognostic significance for each case. 


\section{Bibliography}

1. Davidson Terence M. "Tumors of the head and neck: Clinical and pathological considerations". In: John G. Batsakis, Williams and Wilkins Co., Baltimore, MD (1979): 217-219.

2. SU HH., et al. "Spindle cell carcinoma of the oral cavity and oropharynx: factors affecting outcome". Journal of the Chinese Medical Association 69 (2006): 478-483.

3. Cardesa A and Zidar N. "World Health Organization Classification of Tumors. Head and Neck Tumours: Oral Cavity and Oropharynx". IARC Press, Lyon (2005): 127-128.

4. Anderson CE and Al-Nafussi A. "Spindle cell lesions of the head and neck: an overview and diagnostic approach". Diagnostic Histopathology 15.5 (2009): 264-272.

5. Thompson LD., et al. "Spindle cell (sarcomatoid) carcinomas of the larynx: a clinicopathologic study of 187 cases". The American Journal of Surgical Pathology 26 (2002): 153-170.

6. Leventon GS and Evans HL. "Sarcomatoid Squamous Cell Carcinoma of the Mucous Membranes of the Head and Neck: A Clinicopathologic Study of 20 Cases". Cancer 48 (1981): 994-1003.

7. Thompson LDR. "Squamous cell carcinoma variants of the head and neck". [1] Current Diagnostic Pathology 9 (2003): 384-396.

8. Ramamurti A., et al. "Spindle cell carcinoma of the gingiva: A rare occurrence". Contemporary Clinical Dentistry 4.4 (2013): 500-503.

9. Viswanathan S., et al. "[5] Sarcomatoid (spindle cell) carcinoma of the head and neck mucosal region: a clinicopathologic review of 103 cases from a tertiary referral cancer centre". Head and Neck Pathology 4.4 (2010): 265-275.

10. Feng L., et al. "Spindle cell carcinoma: the general demographics, basic clinico-pathologic characteristics, treatment, outcome and prognostic factors". Oncotarget 8.26 (2017): 43228-43236.

11. Rizzardi C., et al. "A look at the biology of spindle cell squamous carcinoma of the oral cavity: report of a case". Journal of Oral and Maxillofacial Surgery 61 (2003): 264-268.

12. Huang SF., et al. "Sarcomatoid carcinoma of the parotid gland with apparent metastasis of epidermoid elements to cervical lymph nodes". Acta Otolaryngology 126 (2006): 667-671.

13. Altrabulsi B., et al. "Spindle basaloid squamous carcinoma of the upper aerodigestive tract: immunohistochemical and clinicopathological study of three cases". Annals of Diagnostic Pathology 10 (2006): 149-153.

14. Ellis GL., et al. "Spindle-cell carcinoma in the upper aerodigestive tract. An immunohistochemical analysis of 21 cases". The American Journal of Surgical Pathology 11 (1987): 335-342.

15. Munakata R., et al. "Spindle cell carcinoma of the gingiva: report of an autopsy case”. Journal of Oral Pathology and Medicine 27.4 (1998): 180-184.

16. Katase N., et al. "A spindle cell carcinoma presenting with osseous metaplasia in the gingiva: a case report with immunohistochemical analysis". Head and Face Medicine 4 (2008): 28.

17. Kwon GY., et al. "Sarcomatoid carcinoma of the mandible-report of a case". Journal of the Korean Association of Oral and Maxillofacial Surgeons 36 (2010): 228-230.

18. Parikh N and Desai N. "Spindle Cell Carcinoma of the oral cavity: A case report of a rare entity and review of literature". Journal of Advanced Oral Research: SAGE Journals 2 (2011): 3136.

19. Ravindran R., et al. "Spindle cell carcinoma of maxilla: case report of a rare entity and review of literature". Oral and Maxillofacial Pathologists Journal 4.2 (2013): 379-384.

20. Shen $X$ and Liu F. "Primary sarcomatoid carcinoma of the mandibular gingiva: clinicopathological and radiological findings". Singapore Medical Journal 55.9 (2014): 152-155.

21. Shetty V., et al. "Sarcomatoid Carcinoma of Anterior Maxilla: A Case Report and Immunohistochemical Analysis". Journal of Clinical and Diagnostic Research 9.5 (2015): 19-20.

22. Mahajan A., et al. "Sarcomatoid Carcinoma of the Oral Cavity: A Diagnostic Dilemma". Case Reports in Dentistry (2017): 7495695.

23. Patankar S., et al. "Spindle Cell Carcinoma of the Mandibular Gingiva - A Case Report". Journal of Clinical and Diagnostic Research 10.2 (2016): 08-10. 
24. Mathew A. "A Case Report on Rare Entity: Spindle Cell Carcinoma of Maxillary Alveolus". International Journal of Dental Science And Innovative Research 2.3 (2009): 174-117.

25. Palla B., et al. "Spindle cell variant squamous cell carcinoma of the oral cavity: Case presentation and review of literature". Oral and Maxillofacial Surgery Cases 6.3 (2020): 100174.

26. Ono S., et al. "A Case Report of Spindle Cell Carcinoma with Osteoid and Cartilage Formation in the Tongue". Reports 4.1 (2021): 5 .

27. Bravi F., et al. "Foods, nutrients and the risk of oral and pharyngeal cancer". British Journal of Cancer 109.11 (2013): 29042910.

Volume 5 Issue 7 July 2021

(C) All rights are reserved by Flora Verma., et al. 